\title{
ON GEROCH'S COUNTEREXAMPLE TO THE ALGEBRAIC HOPF CONJECTURE
}

\author{
PAUL F. KLEMBECK
}

\begin{abstract}
In this paper we present a simplified version of Geroch's counterexample to the algebraic Hopf conjecture which requires only a knowledge of the formal symmetries of Riemannian curvature tensors.
\end{abstract}

A number of questions in differential geometry have revolved around the algebraic Hopf conjecture: that in even dimensions, a curvature tensor with positive sectional curvatures has positive Gauss-Bonnet integrand. In dimension two the conjecture is trivial, and in dimension four has been proved by Milnor, see [1]. However, in dimension six and higher even dimensions, the conjecture has been shown to be false by Geroch [2]. Geroch's counterexample consists of an abstract algebraic construction, whose understanding requires careful thought. The purpose of this note is to provide an easily understood numerical example which is derived from Geroch's example.

We define the components of a curvature tensor $R$ on a six dimensional vector space $V$ with basis $\left\{e_{1} e_{2} e_{3} e_{4} e_{5} e_{6}\right\}$ by

$$
\begin{aligned}
& R_{1234}=R_{1256}=R_{3456}=R_{1423}=R_{1625}=R_{3645}=1, \\
& R_{1313}=R_{1515}=R_{2424}=R_{2626}=R_{3535}=R_{4646}=3, \\
& R_{1324}=R_{1526}=R_{3546}=2
\end{aligned}
$$

with all components not derivable from these by the symmetry relations $R_{a b c d}=-R_{b a c d}=-R_{a b d c}=R_{c d a b}$ set to zero. It is trivial to check the Bianchi identities and thereby verify that $R$ is indeed a curvature tensor.

One may check that

$$
\begin{aligned}
\sum_{i l k h} a_{i} b_{l} a_{k} b_{h} R_{i l k h}=3\left[\left(a_{1} b_{3}-a_{3} b_{1}\right.\right. & \left.+a_{2} b_{4}-a_{4} b_{2}\right)^{2} \\
+\left(a_{1} b_{5}-a_{5} b_{1}\right. & \left.+a_{2} b_{6}-a_{6} b_{2}\right)^{2} \\
& \left.+\left(a_{3} b_{5}-a_{5} b_{3}+a_{4} b_{6}-a_{6} b_{4}\right)^{2}\right]
\end{aligned}
$$

so all sectional curvatures are nonnegative.

The Gauss-Bonnet integrand is

$$
\Omega=\sum_{i j} \varepsilon(i) \varepsilon(j) R_{i(1) i(2) j(1) j(2)} R_{i(3) i(4) j(3) j(4)} R_{i(5) i(6) j(5) j(6)}
$$

where $i$ and $j$ are permutations and $\varepsilon$ denotes their sign.

Received by the editors July 7, 1975.

AMS (MOS) subject classifications (1970). Primary 53A45, 53C20.

Key words and phrases. Algebraic Hopf conjecture, Hopf conjecture, Gauss-Bonnet integrand. 
For the particular tensor $R$, previously defined, one may check that the only nonzero terms in $\Omega$ are:

$$
\begin{aligned}
& \varepsilon(132645) \varepsilon(241536) R_{1324} R_{2615} R_{4536}=-4, \\
& \varepsilon(134625) \varepsilon(243516) R_{1324} R_{4635} R_{2516}=-4, \\
& \varepsilon(154623) \varepsilon(263514) R_{1526} R_{4635} R_{2314}=-4, \\
& \varepsilon(125634) \varepsilon(341256) R_{1234} R_{5612} R_{3456}=1,
\end{aligned}
$$

and

$$
\varepsilon(142536) \varepsilon(231645) R_{1423} R_{2516} R_{3645}=-1,
$$

and the terms gotten from these by the $2^{6}$ symmetries like

$$
\varepsilon(\text { abcdef }) \varepsilon(\text { ghijkl }) R_{a b g h} R_{c d i j} R_{e f k l}=\varepsilon(\text { bacdef }) \varepsilon(\text { ghijkl }) R_{b a g h} R_{c d i j} R_{e f k l}
$$

(i.e., interchange of the 1, 2 or 3, 4 subscripts of one of the $R$ 's) and the 3 ! symmetries like

$$
\varepsilon(a b c d e f) \varepsilon(\text { ghijkl }) R_{a b g h} R_{c d i j} R_{e f k l}=\varepsilon(\text { cdabef }) \varepsilon(i j g h k l) R_{c d i j} R_{a b g h} R_{e f k l}
$$

(i.e. a permutation of subscripts which interchanges two $R$ 's). Thus the Gauss-Bonnet integrand is $2^{6} \cdot 3 !(-12)<0$.

Thus $R$ is a nonnegative curvature tensor with strictly negative GaussBonnet integrand. If we add to $R$ a positive curvature tensor with small enough components, we will thus produce a positive curvature tensor with strictly negative Gauss-Bonnet integrand.

For higher even dimensions if we extend $R$ by setting $R_{7878}=1$ and $R_{i j k l}=0$ if $i j k l$ contains both a 7 or 8 and something smaller, we also obtain counterexamples to the algebraic Hopf conjecture, in all even dimensions.

It remains an open question whether there is a compact Riemannian manifold with positive sectional curvature but everywhere negative GaussBonnet integrand. However it is always possible to define a metric in a neighborhood of a fixed point $p$ of a manifold having any specified curvature tensor at $p$, and even having an arbitrary number of specified covariant derivatives at $p$ (Klembeck [3]). Thus the counterexample to the algebraic Hopf conjecture provides a local counterexample to the full Hopf conjecture. It may be of interest to note that the metric

$$
g_{i j}=\left(\begin{array}{cccccc}
1-3 x_{3}^{2} & -2 x_{3} x_{4} & 0 & 0 & 0 & 2 x_{2} x_{5} \\
-2 x_{3} x_{4} & 1-3 x_{4}^{2} & 2 x_{1} x_{4} & 0 & 0 & 0 \\
0 & 2 x_{1} x_{4} & 1-3 x_{5}^{2} & -2 x_{5} x_{6} & 0 & 0 \\
0 & 0 & -2 x_{5} x_{6} & 1-3 x_{6}^{2} & 2 x_{3} x_{6} & 0 \\
0 & 0 & 0 & 2 x_{3} x_{6} & 1-3 x_{1}^{2} & -2 x_{1} x_{2} \\
2 x_{2} x_{5} & 0 & 0 & 0 & -2 x_{1} x_{2} & 1-3 x_{2}^{2}
\end{array}\right)
$$

gives the previous $R$ as its curvature tensor at the origin. It is also of interest to note that the process for finding a metric to produce a given curvature at a given point also works for metrics of any prescribed signature. 


\section{REFERENCES}

1. R. L. Bishop and S. I. Goldberg, Some implications of the generalized Gauss-Bonnet theorem, Trans. Amer. Math. Soc. 112 (1964), 508-535. MR 29 \#574.

2. R. Geroch, Positive sectional curvature does not imply positive Gauss-Bonnet integrand, Proc. Amer. Math. Soc. 54 (1976), 267-270.

3. P. Klembeck, Algebraic curvatures and curvatures (to appear).

Department of Mathematics, University of California, los Angeles, California 90024

Current address: Department of Mathematics, Princeton University, Princeton, New Jersey 08540 